\title{
Chronic kidney disease: identification and management in primary care
}

\author{
This article was published in the following Dove Press journal: \\ Pragmatic and Observational Research \\ 17 August 2016 \\ Number of times this article has been viewed
}

\author{
Simon DS Fraser' \\ Tom Blakeman ${ }^{2}$ \\ 'Academic Unit of Primary Care \\ and Population Sciences, Faculty \\ of Medicine, Southampton General \\ Hospital, University of Southampton, \\ Southampton, ${ }^{2}$ National Institute \\ for Health Research Collaboration \\ for Leadership in Applied Health \\ Research Greater Manchester, \\ Centre for Primary Care, Institute of \\ Population Health, The University of \\ Manchester, Manchester, UK
}

Correspondence: Simon DS Fraser Academic Unit of Primary Care and Population Sciences, Faculty of Medicine, Southampton General Hospital, University of Southampton, South Academic Block, Tremona Road, Southampton SOI6 6YD, UK Tel +44238I206530 Email s.fraser@soton.ac.uk

\begin{abstract}
Chronic kidney disease (CKD) is an important and common noncommunicable condition globally. In national and international guidelines, $\mathrm{CKD}$ is defined and staged according to measures of kidney function that allow for a degree of risk stratification using commonly available markers. It is often asymptomatic in its early stages, and early detection is important to reduce future risk. The risk of cardiovascular outcomes is greater than the risk of progression to end-stage kidney disease for most people with CKD. CKD also predisposes to acute kidney injury - a major cause of morbidity and mortality worldwide. Although only a small proportion of people with CKD progress to end-stage kidney disease, renal replacement therapy (dialysis or transplantation) represents major costs for health care systems and burden for patients. Efforts in primary care to reduce the risks of cardiovascular disease, acute kidney injury, and progression are therefore required. Monitoring renal function is an important task, and primary care clinicians are well placed to oversee this aspect of care along with the management of modifiable risk factors, particularly blood pressure and proteinuria. Good primary care judgment is also essential in making decisions about referral for specialist nephrology opinion. As CKD commonly occurs alongside other conditions, consideration of comorbidities and patient wishes is important, and primary care clinicians have a key role in coordinating care while adopting a holistic, patient-centered approach and providing continuity. This review aims to summarize the vital role that primary care plays in predialysis CKD care and to outline the main considerations in its identification, monitoring, and clinical management in this context.
\end{abstract}

Keywords: chronic kidney disease, primary care, identification, monitoring, management

\section{Introduction}

Maintenance of kidney health is a global priority. This reflects the vital role that the kidneys play such as filtering blood to maintain fluid and electrolyte balance and remove waste (including the processing of medicines), releasing hormones to control blood pressure (BP) and stimulate red blood cell production (and so reduce the risk of cardiovascular disease and anemia), and activating vitamin $\mathrm{D}$ to maintain bone health.

Chronic kidney disease (CKD) is a common condition that refers to a long-term loss of kidney function. It tends to be diagnosed in the presence of other comorbidities (particularly hypertension, diabetes, and cardiovascular disease), isolated CKD is the exception rather than the rule, and CKD is associated with socioeconomic deprivation. ${ }^{1-3}$ Effective identification and management are necessary in order to prevent CKD progression and cardiovascular events, reduce the risks associated with acute kidney injury (AKI), and improve patient safety and medicines management. Historically, 
it was considered that managing patients with CKD is the responsibility of nephrologists in secondary care settings, but improved understanding of the nature and implications of early stages of the condition mean that primary care clinicians have an essential role to play. ${ }^{4}$ For those working in primary care, an understanding of the condition is required in order to identify people with $\mathrm{CKD}$, undertake appropriate risk stratification, communicate the diagnosis to patients, and work with them toward optimal management and risk reduction. This review is not a clinical guideline, rather it draws upon existing national and international guidelines to summarize the importance of CKD and provide an overview of key aspects in its management in primary care.

\section{Definition and staging of CKD}

$\mathrm{CKD}$ in primary care is commonly asymptomatic, and the exact pathology underlying its development is often unknown (as no renal biopsy is usually performed). It is identified and defined by the presence of an abnormality of kidney structure or function (or both) present for at least 3 months. ${ }^{5,6}$ It is classified by the degree of renal dysfunction, as measured by the estimated glomerular filtration rate ([eGFR] derived from serum creatinine using standard estimating equations) and by the presence or absence of structural kidney abnormality or by other evidence of chronic kidney damage, particularly albuminuria. ${ }^{6}$ The Kidney Disease Improving Global Outcomes (KDIGO) organization has summarized the stages of CKD using a "traffic light" staging system that incorporates both creatinine-based eGFR and albuminuria (Figure 1). ${ }^{6}$ This classification gives five levels of dysfunction defined by eGFR (G1-G5) and three by albuminuria (A1-A3). As such, a person with normal or mildly decreased eGFR (G1 or G2) and little or no albuminuria (A1) is considered at low risk, whereas someone with eGFR $<60 \mathrm{~mL} / \mathrm{min} / 1.73 \mathrm{~m}^{2}$ and a moderate degree of albuminuria is at greater risk. This inclusion of albuminuria (usually measured by urinary albumin-to-creatinine ratio [ACR]) recognizes the independent prognostic importance of both eGFR and albuminuria to
Prognosis of CKD by GFR and albuminuria categories: KDIGO 2012

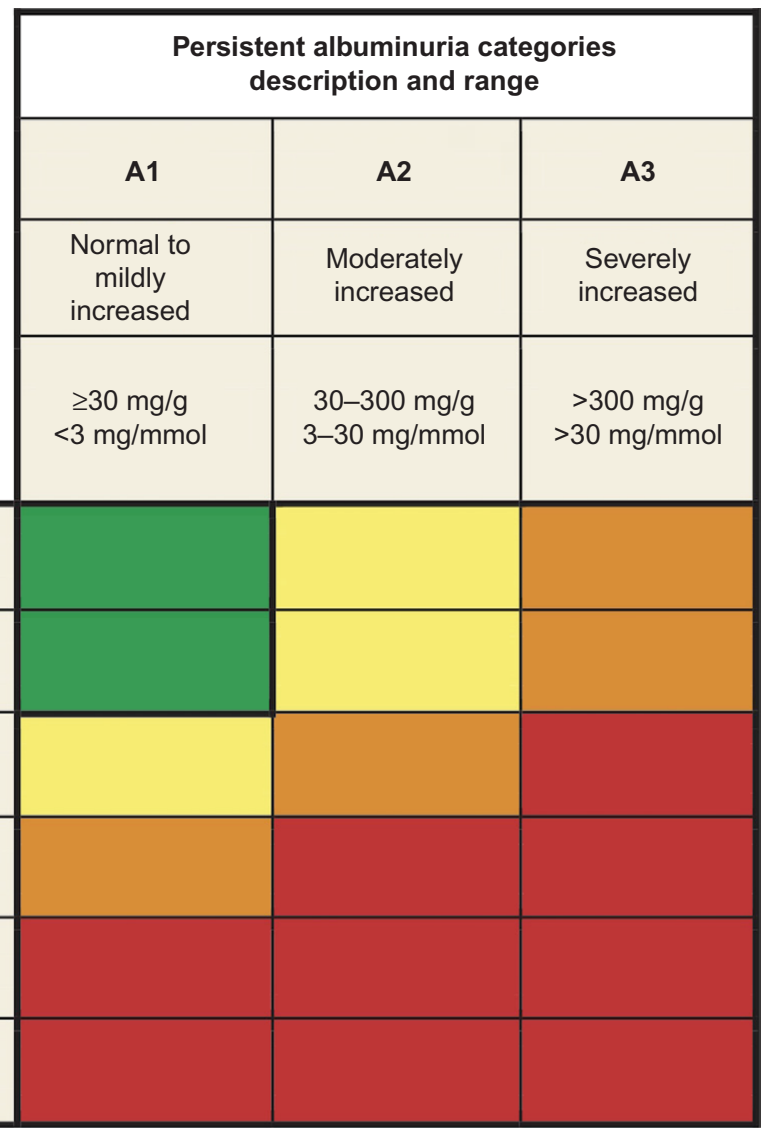

Figure I Prognosis of CKD by GFR and albuminuria category.

Notes: Green: low risk (if no other markers of kidney disease, no CKD); yellow: moderately increased risk; orange: high risk; and red, very high risk. Reprinted by permission from Macmillan Publishers Ltd: Kidney International Supplements. Kidney Disease: Improving Global Outcomes (KDIGO) CKD Work Group. KDIGO 2012 Clinical Practice Guideline for the Evaluation and Management of Chronic Kidney Disease. 20I3;3:I-I50. () 2013 KDIGO. ${ }^{6}$

Abbreviations: CKD, chronic kidney disease; GFR, glomerular filtration rate; KDIGO, Kidney Disease: Improving Global Outcomes. 
a variety of renal and nonrenal outcomes, including mortality, progression to end-stage kidney disease, cardiovascular disease, and AKI. ${ }^{7-14}$ Key to the definition of CKD is the need to assess these markers of renal function over time in order to confirm chronicity.

\section{CKD from a global perspective}

$\mathrm{CKD}$ is an important noncommunicable condition and is a determinant of poor outcomes, often occurring alongside (and worsening the prognosis of) other noncommunicable disease..$^{2-4,15-17}$ The risk of developing cardiovascular disease is greater than the risk of progression to end-stage kidney disease for the majority of people with CKD. ${ }^{11}$ The increased risk of AKI is now being recognized in a major international endeavor to identify and reduce AKI incidence and improve its management. ${ }^{18}$ End-stage kidney disease and consequent need for dialysis or transplantation represent major costs for health care systems and burden for patients, and early detection with primary care intervention to reduce risk of progression is key to sustainability. ${ }^{19-21}$ The financial cost associated with CKD in the UK in 2009-2010 was estimated at $\sim £ 1.45$ billion, or $>1 \%$ of all health service spending in that year, with over half of that spent on renal replacement therapy (RRT), and major cost implications associated with cardiovascular complications. ${ }^{22}$

CKD prevalence is increasing in many countries and was the 18th commonest cause of death globally in the Global Burden of Disease Study 2010 (increased from 27 th in 1990). ${ }^{15,23}$ In developed countries, the prevalence of moderate-to-severe CKD (stages G3-G5) in populationrepresentative surveys is estimated at $5 \%-6 \%$, depending on the equations used to derive eGFR from serum creatinine. ${ }^{24-28}$ Prevalence increases sharply with age and is higher in lower socioeconomic groups and certain ethnic populations. ${ }^{1,24,29-31}$

Despite some controversy over whether people with milder kidney dysfunction should be labeled as having a "disease", the development of international guidelines, such as those from the US National Kidney Foundation Kidney Disease Outcomes Quality Initiative (KDOQI) and KDIGO, represents important developments in terms of the definition of CKD and providing evidence-based recommendations for care, particularly for interventions such as BP control (the highest global risk factor in the Global Burden of Disease Study). ${ }^{632-35}$ Risk factors for the development of CKD include smoking, hypertension, and obesity, all of which are increasing in prevalence and attributable risk status globally with evidence of higher prevalence in lower socioeconomic groups. ${ }^{1,35-38}$

\section{Locations of care}

The predominant location in which care for people with CKD takes place is determined by a number of factors including cause and severity of disease and health system culture, which vary considerably across the world. ${ }^{39}$ Following the instigation of a national renal care framework in the UK $\sim 10$ years ago, and the subsequent incentivization of general practitioners (GPs) to identify and keep registers of people with CKD, the majority of care for people with mild-to-moderate CKD in the UK now occurs in primary care. ${ }^{40,41}$ For patients with more severe kidney disease requiring RRT (dialysis and transplant), the main location of care is usually secondary care. This more severe population is not, therefore, the main focus of this review, although primary care still plays an important supportive role and there is evidence that more guidance is needed for GPs in this area. ${ }^{42}$ There is also mixed evidence about screening for CKD in general populations, which is not discussed in detail, although there is some evidence to support focused screening, for example, in people with diabetes or hypertension, as is currently undertaken as part of the UK National Health Service (NHS) Health Check programme. ${ }^{43-45}$

The rest of this review focuses on the identification of CKD in clinical practice and key aspects of disease management that aim to reduce the risk of adverse outcomes, both renal and nonrenal.

\section{Identifying people with CKD}

Understanding the definition and staging of CKD is crucial to correctly identify people with the condition in clinical practice. Such information is also a key to appropriately advise patients about their kidney health and stratify their future risk.

\section{Diagnostic criteria}

According to the KDIGO CKD guidelines (and the English National Institute for Health and Care Excellence (NICE) CKD guidelines), a patient is identified with CKD if abnormalities of kidney structure or function were present for a minimum of 3 months. ${ }^{5,6}$ The abnormalities are shown in Table 1.

In practice, in primary care, the most important measures to identify CKD are eGFR derived from serum creatinine and ACR derived from a urine sample. NICE recommends that certain populations should be offered testing for CKD using eGFR and ACR (Table 2).

\section{Estimated glomerular filtration rate}

Standardized equations to derive eGFR from serum creatinine are needed because creatinine is an imperfect indicator of 
Table I Diagnostic criteria for CKD

One of the following needs to be present for at least 3 months:

a) Decreased eGFR $\left(<60 \mathrm{~mL} / \mathrm{min} / 1.73 \mathrm{~m}^{2}\right)$

b) One or more marker of kidney damage:

i. Albuminuria (urinary albumin-to-creatinine ratio $[A C R] \geq 30 \mathrm{mg} / \mathrm{g}$ [3 $\mathrm{mg} / \mathrm{mmol}]$ )

ii. Structural abnormalities (from imaging)

iii. Urine sediment abnormalities (hematuria, red or white blood cell casts, oval fat bodies or fatty casts, granular casts, and renal tubular epithelial cells)

iv. Electrolyte and other abnormalities due to tubular disorders

v. Histological abnormalities

vi. Previous history of kidney transplantation

Note: Data from reference 5.

Abbreviations: CKD, chronic kidney disease; eGFR, estimated glomerular filtration rate.

Table 2 People with any of the following risk factors should be offered testing for CKD

- Diabetes

- Hypertension

- Acute kidney injury

- Cardiovascular disease (ischemic heart disease, chronic heart failure, peripheral vascular disease, or cerebral vascular disease)

- Structural renal tract disease, renal calculi, or prostatic hypertrophy

- Multisystem diseases with potential kidney involvement, for example, systemic lupus erythematosus

- Family history of end-stage kidney disease (GFR category G5) or hereditary kidney disease

- Opportunistic detection of hematuria

Note: Data from reference 5.

Abbreviations: CKD, chronic kidney disease; GFR, glomerular filtration rate.

renal excretory function, influenced by other factors (including muscle mass, age, sex, ethnicity, comorbidities, trauma, exercise, and high protein diet). ${ }^{46}$ The common equations in use by laboratories are the modified diet in renal disease and the Chronic Kidney Disease Epidemiology Collaboration (CKDEPI). ${ }^{27,28}$ The CKDEPI is a more accurate equation, and its use in a population tends to reduce the prevalence of CKD but identify a higher risk population. ${ }^{28}$ This has been demonstrated in a large, population-based study in the UK in which a change to the CKDEPI formula was associated with a reduction in overall CKD stage G3-G5 prevalence, but an increase in people older than 70 years and also in the Health Survey for England (which is population representative) in which CKD G3-G5 prevalence was reduced from $6 \%$ to $5.2 \%$ by the use of CKDEPI. ${ }^{25,47}$

Cystatin $\mathrm{C}$ is a further measure of renal function from which eGFR can be derived, less influenced by muscle mass, although it is more expensive and not yet in common use in many locations. ${ }^{48}$

\section{Proteinuria}

There has been considerable debate about different methods of proteinuria identification, including the role of urine dipsticks and protein-to-creatinine ratio. ${ }^{49}$ This has caused some confusion among primary care practitioners. While dipstick tests can detect albumin, they may be less good at detecting other urinary proteins and are poor at protein quantification, and ACR has been shown to have greater sensitivity than protein-to-creatinine ratio for lower levels of proteinuria. ${ }^{50-52}$ There is evidence suggesting that a single ACR, particularly if measured on an early morning urine sample, is a sufficiently sensitive test to identify albuminuria. ${ }^{51}$ These issues were not considered in further detail here, as international guidelines are moving toward a consensus view, and KDIGO now clearly recommends ACR as the investigation of choice and a single early morning urine sample adequate to identify proteinuria. ${ }^{53}$

In clinical practice, although these diagnostic criteria are clear, it can be challenging to apply them. For example, blood and urine tests may need to be repeated in order to identify the chronicity of kidney dysfunction, and the timing of repeat testing needs to be carefully considered. When an eGFR $<60 \mathrm{~mL} / \mathrm{min} / 1.73 \mathrm{~m}^{2}$ is identified in an individual with previously normal renal function, the first step is to confirm the result and to exclude the possibility that the patient is developing transient elevation of creatinine (and fall in eGFR) associated with other factors, such as AKI, by repeat testing within a short period of time. The NICE guidelines recommend "Confirm an eGFR result of $<60 \mathrm{~mL} / \mathrm{min} / 1.73 \mathrm{~m}^{2}$ in a person not previously tested by repeating the test within 2 weeks". ${ }^{5}$ Figure 2 shows a logical diagnostic decision pathway of such an event based on guidelines.

There are therefore a number of important clinical actions to consider when identifying CKD, which depend on a sequence of specific findings. If omissions happen at any stage during this process, for example, failing to repeat test at appropriate time points, failing to check prior records, failing to schedule future blood tests, failing to record the CKD diagnosis appropriately, or failing to communicate the diagnosis with the patient, then diagnosis may not occur and appropriate clinical action may be missed. This can be difficult for primary care organizations operating in a predominantly reactive framework. Adding to this complexity is the need to consider both these eGFR findings and results of urine testing for albuminuria (and potentially other investigations as well such as renal ultrasound). For diagnoses of conditions that do not require consideration of time (eg, making a diagnosis of anemia based on an isolated hemoglobin result) such issues do not usually arise. There is evidence of key clinical actions, such as timely albuminuria testing, being highly dependent on accurate recording of a CKD diagnosis., ${ }^{54}$ The use of software tools to integrate primary care record systems, to 


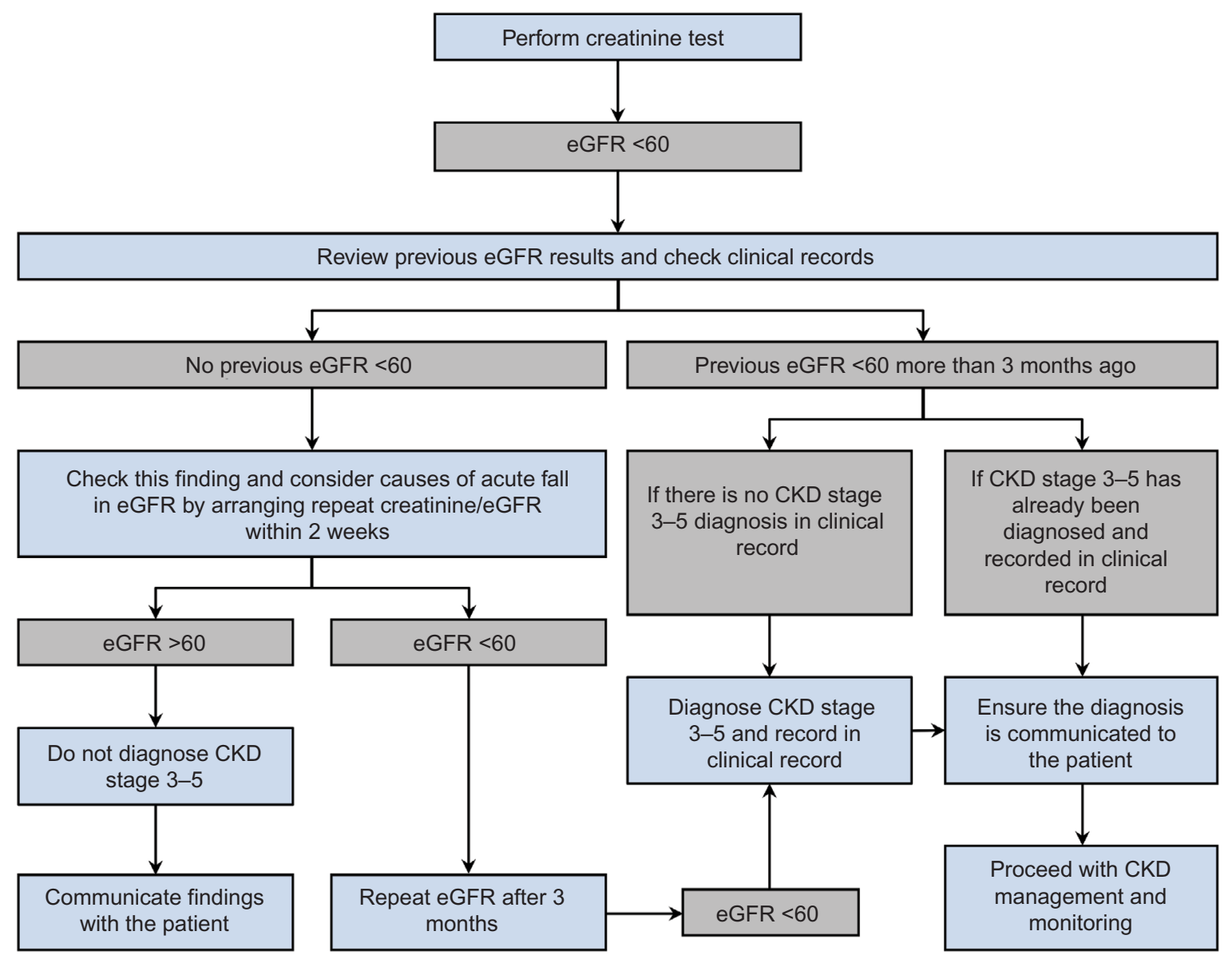

Figure 2 Clinical decision pathway in CKD diagnosis in relation to eGFR.

Notes: Blue boxes represent clinical actions and gray boxes represent findings.

Abbreviations: CKD, chronic kidney disease; eGFR, estimated glomerular filtration rate.

allow regular audit of blood results, and decision support to detect undiagnosed CKD may be one solution. ${ }^{55,56}$

\section{Interpreting test results}

In order to adequately assess renal function, results for both eGFR and ACR are needed and should be interpreted according to KDIGO guidelines (Figure 1). ${ }^{6}$ The most recent NICE guidelines make further refinements to the eGFR/ACR diagnosis of CKD recommended by KDIGO, ${ }^{5,34,57}$ These include the targeted use of cystatin $\mathrm{C}$ in people who are considered "borderline" CKD (ie, people with a creatinine-based eGFR $45-59 \mathrm{~mL} / \mathrm{min} / 1.73 \mathrm{~m}^{2}$ but no evidence of proteinuria). ${ }^{5}$ Use of combinations of markers in this way has been shown to improve risk stratification as discussed below. ${ }^{58,59}$

\section{Disclosing the diagnosis to patients}

Once the diagnosis of CKD is established, an important consideration is how to communicate this with the patient. However, while discussions around CKD and the maintenance of kidney health may be a platform to address both vascular risk and reduce the demands on urgent care through the prevention of AKI, patient and public understanding remains limited. ${ }^{60-63}$ In the UK, a recent national survey indicated that only one in two people are aware that the kidneys make urine and that only $12 \%$ of the population have knowledge that the kidneys have a role in processing medicines. ${ }^{64}$

All disease classification systems have the potential to both structure and constrain the delivery of care, and the introduction of the KDOQI framework for CKD in 2002 is no exception. ${ }^{65}$ There has been considerable controversy around CKD with concerns of over diagnosis and "medicalization of the aging process and the attendant potential for unintended harm. ${ }^{966}$ From a primary care perspective, the literature indicates limited patient awareness of the diagnosis of CKD as well as difficulties surrounding communication, with GPs and nurses reporting reticence to discuss the diagnosis, particularly with elderly patients and those with CKD stage G3a. ${ }^{60,67-69}$

As discussed, a shift to use CKD-EPI formula reduces the prevalence of CKD, particularly at the stages G2-G3 cutoff, and in doing so, may address concerns about unnecessary 
disease labeling and patient monitoring. ${ }^{25,47,66}$ In addition, there are trial-based data demonstrating that it is possible to provide information about CKD without a harmful effect on patient anxiety. ${ }^{70}$ To date, management of early disease has largely focused on tight management of BP, and although in the UK NICE guidance refers to target ranges, concerns have been raised about the "risk of bringing BP to excessively low levels", particularly in the elderly. ${ }^{66}$ However, there may be benefits in framing CKD as a "barometer of overall health". ${ }^{71}$ With the increased recognition in the importance of the association between CKD and risk of AKI, there are now resources to broaden and tailor conversations with patients in order to support management of episodes of acute illness in addition to addressing vascular risk. ${ }^{72}$

\section{Managing people with CKD}

Primary care plays an important role in the monitoring and management of CKD, particularly in efforts to reduce the risk of cardiovascular disease and other complications. Only a small proportion of people with mild or moderate CKD will progress to end-stage disease. In a populationbased study in Norway (the HUNT II study), for example, of 3,069 people with CKD followed for median 8 years, only $38(1 \%)$ progressed to end-stage disease. ${ }^{44}$ Therefore, the main purpose of monitoring in people with CKD is to undertake risk stratification incorporating objective measures alongside clinical judgment in discussion with the patient and to regularly review this risk, not only to identify this small proportion of people at risk of disease progression but also to identify those at risk of other complications, particularly cardiovascular disease and AKI.

\section{CKD progression}

A great deal of scientific endeavor has focused on trying to identify those people with CKD who are at risk of progression. This has included optimizing the use of existing biomarkers, alone and in combination. Peralta et al ${ }^{58}$ showed that combining creatinine-defined eGFR with ACR and cystatin C-derived eGFR improved the ability to correctly identify those at greater risk of progression and mortality. Several risk scores have been developed (23 different models identified in a recent systematic review), most notably, from the perspective of the broad population of people with CKD, developed by Tangri et al, ${ }^{73,74}$ which included commonly used demographic and clinical markers to identify people at risk of progression. Other novel risk factors have also been explored, including genetic markers, fibroblast growth factor 23 and new cardiovascular risk factors (CXC motif, ligand 12, and ceruloplasmin), skin autofluorescence, and other inflammatory markers (serumfree light chains and circulating receptors for tumor necrosis factor). ${ }^{75}$ It is beyond the remit of this review to describe all of these in detail, and we instead focus on the role of measures in common use in clinical practice, particularly eGFR, ACR, and BP.

\section{Estimated glomerular filtration rate}

There has been debate about creatinine testing frequency for monitoring eGFR, and recommendations are based more on expert opinion than formal research evidence. The frequencies recommended by NICE and KDIGO therefore differ slightly, but those from KDIGO are set out in Table 3 along with the KDIGO color scheme to reflect risk of progression in each category. ${ }^{5,6}$

KDIGO guidelines recommend testing with a slightly greater frequency than NICE such as three tests per year for people with CKD stage G4A2 rather than twice a year recommended by $\mathrm{NICE}^{5,6}$

Progression of CKD is defined in the most recent NICE guidelines as a sustained decrease in eGFR of $25 \%$ or more and a change in GFR category within 12 months or a sustained decrease in GFR of $15 \mathrm{~mL} / \mathrm{min} / 1.73 \mathrm{~m}^{2}$ per year. $^{5}$ NICE also recommends that rate of progression should be identified using three GFR estimations over at least 90 days.

\section{Proteinuria}

There is strong evidence from international meta-analyses of large-scale general population cohorts of the independent predictive ability of ACR measurement for a wide variety of adverse clinical outcomes including all-cause and

Table 3 Recommended annual frequency of eGFR testing in people with CKD (number of tests)

\begin{tabular}{llll}
\hline eGFR categories $(\mathbf{m L} /$ & \multicolumn{3}{l}{ ACR categories $(\mathbf{m g} / \mathbf{m m o l})$} \\
\cline { 2 - 4 } $\left.\mathbf{m i n} / \mathbf{I . 7 3} \mathbf{~ m}^{\mathbf{2}}\right)$ & AI $(<3)$ & A2 $(\mathbf{3 - 3 0})$ & A3 (>30) \\
\hline GI $(\geq 90)$ & I if CKD & I & 2 \\
G2 (60-89) & I if CKD & I & 2 \\
G3a (45-59) & I & 2 & 3 \\
G3b (30-44) & 2 & 3 & 3 \\
G4 (I5-29) & 3 & 3 & $4+$ \\
G5 (<15) & $4+$ & $4+$ & $4+$
\end{tabular}

Notes: Green: low risk (if no other markers of kidney disease, no CKD); yellow: moderately increased risk; orange: high risk; and red: very high risk. Reprinted by permission from Macmillan Publishers Ltd: Kidney International Supplements. Kidney Disease: Improving Global Outcomes (KDIGO) CKD Work Group. KDIGO 2012 Clinical Practice Guideline for the Evaluation and Management of Chronic Kidney Disease. 2013;3:1-150. ${ }^{6}$

Abbreviations: eGFR, estimated glomerular filtration rate; CKD, chronic kidney disease; ACR, albumin-to-creatinine ratio; KDIGO, Kidney Disease: Improving Global Outcomes. 
cardiovascular mortality, AKI, CKD progression, and heartfailure-related hospitalization. ${ }^{8,11,76-78}$

Recommended frequency of ACR monitoring also varies across different guidelines. Johnson ${ }^{49}$ provided a useful summary of the variation, with several bodies not specifying a recommended testing frequency (the KDOQI, Caring for Australasians with Renal Insufficiency [CARI], Scottish Intercollegiate Guidelines Network [SIGN], and Canadian Society of Nephrology [CSN]), while others recommend annually or "at least annually" (NICE, Royal Australian College of General Practitioners [RACGP], and European Renal Best Practice Guidelines [ERBP]). KDIGO guidelines recognize the areas of uncertainty in this area: "We recommend further research to more accurately define the frequency with which GFR and albuminuria measurements should be performed based on their ability to inform strategies which prevent adverse outcomes (eg, progression of kidney disease and death)". ${ }^{6}$ This therefore remains an area for which further evidence is needed, and, in the meantime, frequency of monitoring should be guided by the clinical situation and degree of risk as stratified using the KDIGO framework. For the majority of people with CKD in primary care (who are likely to have stages G1-G3 and A1), annual ACR testing is likely to be sufficient to quantify the degree of any albuminuria and detect change. ${ }^{5}$ This is best conducted on an early morning urine specimen, particularly for quantification. ${ }^{5}$

\section{Blood pressure}

Managing hypertension is a key strength of primary care and controlling BP arguably the most important intervention in reducing both progression and cardiovascular risk in people with CKD. A very high proportion of the population of people with CKD have hypertension. In a large database study in Canada $(n=530,771), \sim 47 \%$ of people with all stages CKD had hypertension. ${ }^{3}$ In the US Kidney Early Evaluation Program (KEEP) database, hypertension prevalence was $>80 \%$ among people with eGFR $<60 \mathrm{~mL} /$ $\mathrm{min} / 1.73 \mathrm{~m}^{2}$ or albuminuria $\geq 3 \mathrm{mg} / \mathrm{mmol}$, and in a UK cohort study of 1,741 people with CKD stage 3 , the prevalence was $88 \%$. $^{79,80}$

Tight control of systolic BP is associated with reduced risk of progression and cardiovascular disease in people with CKD. ${ }^{81}$ A variety of BP targets have been recommended, which can be confusing for clinicians. ${ }^{5,33,34}$ Moreover, CKD often occurs with other chronic conditions (particularly hypertension and diabetes) causing further uncertainty about which set of chronic disease guidelines should be followed with respect to BP control. Evidence from a cohort study of people with moderate CKD in the UK has shown that
BP control often fails to meet guideline levels, particularly the more stringent targets set for people with diabetes or albuminuria. ${ }^{79}$ This can mean that BP control is often not achieved in those for whom it is most important in terms of reducing future risk.

KDIGO guidelines for managing BP in CKD advise individualizing BP targets according to age and presence of other comorbidities but recommend the same targets for people with and without diabetes. ${ }^{34}$ Their recommendations for BP control among people with nondialysis dependent CKD are shown in Table 4. NICE guidelines provide a target range with similar thresholds. ${ }^{5}$

The potential adverse effects of achieving too low BP in people with $\mathrm{CKD}$ have raised questions about the role of such targets and clinical judgment should also be applied. ${ }^{5,82}$ Similarly, the optimal frequency of BP monitoring is guided more by individual clinical circumstances. Audit-based education is a useful method to support BP monitoring efforts, augment guidelines, and help clinicians achieve systolic BP targets in people with CKD. ${ }^{83}$ Remote monitoring is an emerging area that may have a role to play among the large population of people with CKD and hypertension. ${ }^{84}$

Controlling BP may require both lifestyle and pharmaceutical intervention. Lifestyle recommendations include achieving and maintaining a healthy weight (body mass index 20-25), lowering salt intake to $<90 \mathrm{mmol}(2 \mathrm{~g}) / \mathrm{d}$ of sodium, undertaking exercise compatible with cardiovascular health (aiming for 30 minutes five times a week), and limiting alcohol to no more than two standard drinks per day for men and one for women. ${ }^{34}$

KDIGO generally considers achieving optimal level of control of BP to be more important that specific choice of antihypertensive. ${ }^{34}$ Choice should therefore take a holistic view and be aligned with clinical judgment and with general hypertension guidelines. There are, however, specific circumstances in which renin-angiotensin-aldosterone system inhibitors (including angiotensin-converting enzyme inhibitors, angiotensin receptor blockers, direct renin inhibitors, and aldosterone antagonists) should be used. Reducing proteinuria, for

Table 4 KDIGO recommended BP targets for people with CKD

\begin{tabular}{lllll}
\hline Diabetes status & BP category & \multicolumn{3}{l}{ ACR categories $(\mathbf{m g} / \mathbf{m m o l})$} \\
\cline { 2 - 5 } & & Al $(<3)$ & A2 (3-30) & A3 (>30) \\
\hline Diabetes & Systolic & $\leq 140$ & $\leq 130$ & $\leq 130$ \\
& Diastolic & $\leq 90$ & $\leq 80$ & $\leq 80$ \\
No diabetes & Systolic & $\leq 140$ & $\leq 130$ & $\leq 130$ \\
& Diastolic & $\leq 90$ & $\leq 80$ & $\leq 80$ \\
\hline
\end{tabular}

Note: Data from reference. ${ }^{34}$

Abbreviations: KDIGO, Kidney Disease Improving Global Outcomes; BP, blood pressure; CKD, chronic kidney disease; $A C R$, albumin-to-creatinine ratio. 
Table 5 People with CKD and the following should be offered a rennin-angiotensin-aldosterone system inhibitor

- Diabetes and an ACR of $\geq 3 \mathrm{mg} / \mathrm{mmol}$ (ACR category A2 or A3)

- Hypertension and an ACR of $\geq 30 \mathrm{mg} / \mathrm{mmol}$ (ACR category A3)

- An ACR of $\geq 70 \mathrm{mg} / \mathrm{mmol}$ (irrespective of hypertension or cardiovascular disease)

Note: Data from reference 5.

Abbreviations: CKD, chronic kidney disease; $A C R$, albumin-to-creatinine ratio.

example, is an important goal in reducing future risk. These are set out (from NICE guidelines) in Table 5.

NICE recommends that people treated with renin-angiotensin-aldosterone system inhibitors should be informed that achieving the optimal tolerated dose and monitoring eGFR and serum potassium to achieve this safely are important. ${ }^{5}$ In recognition of the potential problems associated with overtreatment of BP, particularly among the predominantly older population with CKD and comorbidities, KDIGO recommends that treatments should be tailored with "gradual escalation of treatment and close attention to adverse events related to BP treatment, including electrolyte disorders, acute deterioration in kidney function, orthostatic hypotension and drug side effects" ${ }^{34}$ Both KDIGO and NICE advise against using combined angiotensin-converting enzyme and angiotensin receptor blocker in people with CKD due to insufficient evidence of benefit. ${ }^{5,6}$ Research currently underway will help with uncertainties in the role of aldosterone receptor antagonism in $\mathrm{CKD} .{ }^{85}$

\section{Maintenance of vascular health and cardiovascular risk reduction}

Many of the efforts aimed at reducing progression risk, such as lifestyle interventions and BP control, have dual benefits in cardiovascular risk reduction. Indeed, there has been debate about whether CKD indices should be added to existing cardiovascular risk scores. ${ }^{86}$ An attempt has been made to do this in QRisk2®, for example, which includes a "yes/no" option for CKD stage 4 or 5 as part of its algorithm. ${ }^{87}$ This is a valuable addition, but it is worth remembering that the relationships between eGFR and ACR and cardiovascular disease appear to be continuous, and therefore, as recognized by KDIGO, people with any degree of CKD should be considered at increased cardiovascular risk compared with the general population. ${ }^{6,11,78}$ NICE recommends that lipid modification guidelines should be followed for the use of statins in people with CKD, which recommends use of atorvastatin $20 \mathrm{mg}$ for primary or secondary prevention. ${ }^{5,88}$ Both NICE and KDIGO recommend offering antiplatelet drugs to people with CKD for the secondary prevention of cardiovascular disease but with awareness of the increased risk of bleeding that needs to be balanced against the possible cardiovascular benefits. ${ }^{6,88}$

\section{Patient safety, reducing AKI risk, and optimizing medicines management}

An important emerging role for primary care is in the prevention of AKI. It is estimated that about one in five emergency hospital admissions from primary care may be associated with AKI ${ }^{89}$ Key considerations include identifying those at risk (CKD, sepsis, dehydration, and hypovolemia), good medicines management (avoiding nonsteroidal anti-inflammatory drugs and other nephrotoxic agents), administration of key immunizations to reduce infection risk (including influenza and pneumococcal), and identifying those with deteriorating renal function by serum creatinine testing..$^{90}$ It is also important to monitor for evidence of CKD progression post-AKI, including regular review of medication.

A key issue is to recognize that CKD affects the metabolism of medications and, conversely, many medications can affect renal function.

In early CKD, comorbidities may be of greater importance to patients with CKD, although this may change if CKD progresses. ${ }^{91}$ Clinicians in primary care therefore have an important overseeing and coordination role to provide better integration of care for people with CKD. ${ }^{92}$ Linked to this is the importance of good communication with patients who have CKD. This applies at mild-to-moderate stages in order to discuss risk stratification and inform self-management efforts including smoking cessation and weight loss (if overweight or obese) and the potential need for pharmacological intervention. It is also vital in more advanced CKD where informed decisions are needed with regard to dialysis and transplantation and in decisions to opt for palliative treatment. ${ }^{93}$ In this context, the evidence that health literacy may be poor in a high proportion of people with CKD is of concern. ${ }^{94}$ The response to this, however, may not just be to simplify messages for patients but to encourage education programmes and patient support groups in order to empower patients and increase self-efficacy.

\section{Making decisions about referral}

Deciding when to refer a patient with CKD for specialist opinion can be difficult. Consideration of a number of factors is required, including consideration of the extent and impact of comorbidities, and the patient's wishes should be a prominent factor. NICE guidance sets out some referral criteria for consideration. These are shown in Table 6. 
Table 6 Nephrology referral should be considered for the following people with CKD

- GFR $<30 \mathrm{~mL} / \mathrm{min} / 1.73 \mathrm{~m}^{2}$ (GFR category G4 or G5), with or without diabetes

- $A C R \geq 70 \mathrm{mg} / \mathrm{mmol}$, unless known to be caused by diabetes and already appropriately treated

- $A C R \geq 30 \mathrm{mg} / \mathrm{mmol}$ (ACR category $A 3$ ), together with hematuria

- Sustained decrease in GFR of $\geq 25 \%$, and a change in GFR category or sustained decrease in GFR of $\geq 15 \mathrm{~mL} / \mathrm{min} / 1.73 \mathrm{~m}^{2}$ within 12 months

- Hypertension that remains poorly controlled despite the use of at least four antihypertensive drugs at therapeutic dose

- Known or suspected rare or genetic causes of CKD

- Suspected renal artery stenosis

Note: Data from reference 5 .

Abbreviations: CKD, chronic kidney disease; GFR, glomerular filtration rate; ACR, albumin-to-creatinine ratio.

However, this should not be considered an exhaustive list; other bodies' recommendations have included AKI, recurrent nephrolithiasis, and suspected renal anemia as indications for referral, for example. ${ }^{6}$ Advice may also be needed at earlier stages of CKD. In a cross-sectional study exploring treatment needs of 1,741 people with CKD stage 3 in the UK, $>30 \%$ required improved BP control, $8 \%$ required investigations for anemia, $>7 \%$ needed to stop potentially nephrotoxic drugs, and $\sim 6 \%$ required referral to a nephrology specialist. ${ }^{95}$ Reasons for needing nephrology referral included GFR decline/progression to CKD 4 or 5 , proteinuria, and complications of CKD. ${ }^{95}$

\section{Quality improvement}

Given these multiple roles that primary care plays in CKD, it is important to identify evidence for interventions aimed at improving quality of care. A useful summary is given by Taal, which summarizes the evidence for interventions such as eGFR reporting, enhanced prompts with eGFR, the role of pay for performance, audit-based education, and telemedicine. ${ }^{96}$ Features associated with successful implementation of CKD interventions in primary care include: framing CKD in the context of general well-being and vascular health as well as in terms of patient safety and medicines management; ensuring compatibility with existing clinical practice or with patient's everyday lives; and enabling ownership of feedback processes to create individualized improvements. ${ }^{97}$ Other important considerations are clear mechanisms for CKD detection in those at risk as well as appropriate training for health practitioners, particularly in parts of the world where primary care may be less established but where CKD prevalence and impact are increasing. ${ }^{98}$

\section{Conclusion}

$\mathrm{CKD}$ is a common condition, the prevalence of which is likely to increase globally with aging populations and growing prevalence of risk factors such as obesity. CKD has important prognostic implications, and many health systems cannot afford to see increasing numbers of people progress to endstage renal disease and require dialysis or renal transplant. CKD is often asymptomatic in its early stages, and clinicians working in primary care have a vital role to play in its identification, risk stratification, and monitoring. Primary care also has a pivotal role in the prevention of complications and progression in managing risk factors such as high $\mathrm{BP}$ and the prevention in AKI. CKD often occurs in conjunction with other chronic disease comorbidities, and primary care clinicians are best placed to take a holistic view of care in mild-to-moderate CKD and empower patients. In alignment with the principles of the World Health Organisation World Health Report 2008, CKD is a good exemplar of why primary health care is needed now more than ever. ${ }^{99}$

\section{Acknowledgments}

Simon DS Fraser is funded by the National Institute for Health Research. Tom Blakeman is funded by the National Institute for Health Research Collaboration for Leadership in Applied Health Research and Care (NIHR CLAHRC) Greater Manchester. The funders had no role in the preparation of this document. However, the work outlined in this document may be considered to be affiliated to the work of the NIHR CLAHRC Greater Manchester. The views expressed in this document are those of the authors and not necessarily those of the NHS, NIHR, or the Department of Health.

\section{Disclosure}

The authors report no conflicts of interest in this work.

\section{References}

1. Fraser SD, Roderick PJ, Aitken G, et al. Chronic kidney disease, albuminuria and socioeconomic status in the Health Surveys for England 2009 and 2010. J Public Health (Oxf). 2014;36(4):577-586.

2. Fraser SD, Roderick PJ, May C, et al. The burden of comorbidity in people with chronic kidney disease stage 3: a cohort study. BMC Nephrol. $2015 ; 16: 193$.

3. Tonelli M, Wiebe N, Guthrie B, et al. Comorbidity as a driver of adverse outcomes in people with chronic kidney disease. Kidney Int. 2015;88(4): 859-866.

4. Eckardt KU, Coresh J, Devuyst O, et al. Evolving importance of kidney disease: from subspecialty to global health burden. Lancet. 2013;382(9887): 158-169.

5. NICE Clinical Guidelines 182. Chronic Kidney Disease: Early Identification and Management of Chronic Kidney Disease in Adults in Primary and Secondary Care. London: National Institute of Health and Care Excellence; 2014.

6. Kidney Disease: Improving Global Outcomes (KDIGO) CKD Work Group. KDIGO 2012 Clinical Practice Guideline for the Evaluation and Management of Chronic Kidney Disease. Kidney International Supplements. 2013;3:1-150. 
7. Arnlov J, Evans JC, Meigs JB, et al. Low-grade albuminuria and incidence of cardiovascular disease events in nonhypertensive and nondiabetic individuals - The Framingham heart study. Circulation. 2005;112(7):969-975.

8. Gansevoort RT, Matsushita K, van der Velde M, et al; Chronic Kidney Disease Prognosis Consortium. Lower estimated GFR and higher albuminuria are associated with adverse kidney outcomes. A collaborative meta-analysis of general and high-risk population cohorts. Kidney Int. 2011;80(1):93-104.

9. Hallan S, Astor B, Romundstad S, Aasarod K, Kvenild K, Coresh J. Association of kidney function and albuminuria with cardiovascular mortality in older vs younger individuals - the HUNT II study. Arch Intern Med. 2007;167(22):2490-2496.

10. Hsu RK, Hsu C-Y. Proteinuria and reduced glomerular filtration rate as risk factors for acute kidney injury. Curr Opin Nephrol Hypertens. 2011;20(3):211-217.

11. Chronic Kidney Disease Prognosis Consortium; Matsushita K, van der Velde M, et al. Association of estimated glomerular filtration rate and albuminuria with all-cause and cardiovascular mortality in general population cohorts: a collaborative meta-analysis. Lancet. 2010;375(9731):2073-2081.

12. Nitsch D, Grams M, Sang Y, et al; Chronic Kidney Disease Prognosis Consortium. Associations of estimated glomerular filtration rate and albuminuria with mortality and renal failure by sex: a meta-analysis. BMJ. 2013;346:f324.

13. Grams ME, Astor BC, Bash LD, Matsushita K, Wang Y, Coresh J. Albuminuria and estimated glomerular filtration rate independently associate with acute kidney injury. J Am Soc Nephrol. 2010;21(10):1757-1764.

14. Go AS, Chertow GM, Fan DJ, McCulloch CE, Hsu CY. Chronic kidney disease and the risks of death, cardiovascular events, and hospitalization. N Engl J Med. 2004;351(13):1296-1305.

15. Couser WG, Remuzzi G, Mendis S, Tonelli M. The contribution of chronic kidney disease to the global burden of major noncommunicable diseases. Kidney Int. 2011;80(12):1258-1270.

16. Jha V, Garcia-Garcia G, Iseki K, et al. Chronic kidney disease: global dimension and perspectives. Lancet. 2013;382(9888):260-272.

17. Stevens LA, Li S, Wang C, et al. Prevalence of CKD and comorbid illness in elderly patients in the United States: results from the kidney early evaluation program (KEEP). Am J Kidney Dis. 2010;55(3 suppl 2):S23-S33.

18. Mehta RL, Cerda J, Burdmann EA, et al. International Society of Nephrology's 0by 25 initiative for acute kidney injury (zero preventable deaths by 2025): a human rights case for nephrology. Lancet. 2015;385(9987):2616-2643.

19. Meguid El Nahas A, Bello AK. Chronic kidney disease: the global challenge. Lancet. 2005;365(9456):331-340.

20. Grassmann A, Gioberge S, Moeller S, Brown G. ESRD patients in 2004: global overview of patient numbers, treatment modalities and associated trends. Nephrol Dial Transplant. 2005;20(12): $2587-2593$.

21. Radhakrishnan J, Remuzzi G, Saran R, et al. Taming the chronic kidney disease epidemic: a global view of surveillance efforts. Kidney Int. 2014;86(2):246-250.

22. Kerr M, Bray B, Medcalf J, O’Donoghue DJ, Matthews B. Estimating the financial cost of chronic kidney disease to the NHS in England. Nephrol Dial Transplant. 2012;27(suppl 3):iii73-iii80.

23. Lozano R, Naghavi M, Foreman K, et al. Global and regional mortality from 235 causes of death for 20 age groups in 1990 and 2010: a systematic analysis for the Global Burden of Disease Study 2010. Lancet. 2012;380(9859):2095-2128.

24. Coresh J, Astor BC, Greene T, Eknoyan G, Levey AS. Prevalence of chronic kidney disease and decreased kidney function in the adult US population: Third National Health and Nutrition Examination Survey. Am J Kidney Dis. 2003;41(1):1-12.

25. Fraser SD, Aitken G, Taal MW, et al. Exploration of chronic kidney disease prevalence estimates using new measures of kidney function in the health survey for England. PLoS One. 2015;10(2):e0118676.

26. Hallan SI, Coresh J, Astor BC, et al. International comparison of the relationship of chronic kidney disease prevalence and ESRD risk. J Am Soc Nephrol. 2006;17(8):2275-2284.
27. Levey AS, Coresh J, Greene T, et al; Chronic Kidney Disease Epidemiology Collaboration. Using standardized serum creatinine values in the modification of diet in renal disease study equation for estimating glomerular filtration rate. Ann Intern Med. 2006;145(4):247-254.

28. Levey AS, Stevens LA, Schmid CH, et al. A new equation to estimate glomerular filtration rate. Ann Intern Med. 2009;150(9):604-612.

29. Coresh J, Selvin E, Stevens LA, et al. Prevalence of chronic kidney disease in the United States. JAMA. 2007;298(17):2038-2047.

30. Dreyer G, Hull S, Aitken Z, Chesser A, Yaqoob MM. The effect of ethnicity on the prevalence of diabetes and associated chronic kidney disease. QJM. 2009;102(4):261-269.

31. Hossain MP, Palmer D, Goyder E, El Nahas AM. Social deprivation and prevalence of chronic kidney disease in the UK: workload implications for primary care. $Q J M .2012 ; 105(2): 167-175$.

32. Kidney Disease Outcomes Quality Initiative (K/DOQI). K/DOQI clinical practice guidelines for chronic kidney disease: evaluation, classification, and stratification. Am J Kidney Dis. 2002;39(2 suppl 1):S1-S266.

33. Kidney Disease Outcomes Quality Initiative (K/DOQI). K/DOQI clinical practice guidelines on hypertension and antihypertensive agents in chronic kidney disease. Am J Kidney Dis. 2004;43(5 suppl 1):S1-S290.

34. Kidney Disease: Improving Global Outcomes (KDIGO) Blood Pressure Work Group. KDIGO clinical practice guideline for the management of blood pressure in chronic kidney disease. Kidney Int Suppl. 2012;2(5):337-414.

35. Lim SS, Vos T, Flaxman AD, et al. A comparative risk assessment of burden of disease and injury attributable to 67 risk factors and risk factor clusters in 21 regions, 1990-2010: a systematic analysis for the Global Burden of Disease Study 2010. Lancet. 2012;380(9859):2224-2260.

36. Haroun MK, Jaar BG, Hoffman SC, Comstock GW, Klag MJ, Coresh J. Risk factors for chronic kidney disease: a prospective study of 23,534 men and women in Washington County, Maryland. J Am Soc Nephrol. 2003;14(11):2934-2941.

37. Pinto-Sietsma SJ, Navis G, Janssen WMT, et al; PREVEND Study Group. A central body fat distribution is related to renal function impairment, even in lean subjects. Am J Kidney Dis. 2003;41(4):733-741.

38. Ting SM, Nair H, Ching I, Taheri S, Dasgupta I. Overweight, obesity and chronic kidney disease. Nephron Clin Pract. 2009;112(3):c121-c127.

39. White SL, Chadban SJ, Jan S, Chapman JR, Cass A. How can we achieve global equity in provision of renal replacement therapy? Bull World Health Organ. 2008;86(3):229-237.

40. Stevens PE, de Lusignan S, Farmer CK, Tomson CR. Engaging primary care in CKD initiatives: the UK experience. Nephrol Dial Transplant. 2012;27(suppl 3):iii5-iii11.

41. Department of Health. National Service Framework for Renal Services - Part Two: Chronic Kidney Disease, Acute Renal Failure and End of Life Care. London: Department of Health; 2005:30.

42. Tonkin-Crine S, Santer M, Leydon GM, et al. GPs' views on managing advanced chronic kidney disease in primary care: a qualitative study. Br J Gen Pract. 2015;65(636):e469-e477.

43. NHS Health Check Programme [homepage on the Internet]. Available from: http://www.healthcheck.nhs.uk/. Accessed January 29, 2013.

44. Hallan SI, Dahl K, Oien CM, et al. Screening strategies for chronic kidney disease in the general population: follow-up of cross sectional health survey. BMJ. 2006;333(7577):1047.

45. van der Velde M, Halbesma N, de Charro FT, et al. Screening for albuminuria identifies individuals at increased renal risk. J Am Soc Nephrol. 2009;20(4):852-862.

46. Perrone RD, Madias NE, Levey AS. Serum creatinine as an index of renal function: new insights into old concepts. Clin Chem. 1992;38(10): 1933-1953.

47. O'Callaghan CA, Shine B, Lasserson DS. Chronic kidney disease: a large-scale population-based study of the effects of introducing the CKD-EPI formula for eGFR reporting. BMJ Open. 2011;1(2):e000308.

48. Peralta CA, Katz R, Sarnak MJ, et al. Cystatin C identifies chronic kidney disease patients at higher risk for complications. J Am Soc Nephrol. 2011;22(1):147-155.

49. Johnson DW. Global proteinuria guidelines: are we nearly there yet? Clin Biochem Rev. 2011;32(2):89-95. 
50. Browne OT, Bhandari S. Interpreting and investigating proteinuria. BMJ. 2012;344:e2339.

51. Fraser SD, Roderick PJ, McIntyre NJ, et al. Assessment of proteinuria in patients with chronic kidney disease stage 3: albuminuria and nonalbumin proteinuria. PLoS One. 2014;9(5):e98261.

52. White SL, Yu R, Craig JC, Polkinghorne KR, Atkins RC, Chadban SJ. Diagnostic accuracy of urine dipsticks for detection of albuminuria in the general community. Am J Kidney Dis. 2011;58(1):19-28.

53. Lamb EJ, MacKenzie F, Stevens PE. How should proteinuria be detected and measured? Ann Clin Biochem. 2009;46(pt 3):205-217.

54. Fraser S, Parkes J, Culliford D, Santer M, Roderick P. Timeliness in chronic kidney disease and albuminuria identification: a retrospective cohort study. BMC Fam Pract. 2015;16(1):18.

55. Ennis J, Gillen D, Rubenstein A, et al. Clinical decision support improves physician guideline adherence for laboratory monitoring of chronic kidney disease: a matched cohort study. BMC Nephrol. 2015;16:163.

56. Caplin B, Wheeler D, Nitsch D, Hull S. The National Chronic Kidney Disease Audit Pilot Report, June-December 2014. Available from: http:// www.ckdaudit.org.uk/news-announcements/pilot-report\#.V3495fkrLcs. Accessed July 6, 2016.

57. Carville S, Wonderling D, Stevens P; Guideline Development Group. Early identification and management of chronic kidney disease in adults: summary of updated NICE guidance. BMJ. 2014;349:g4507.

58. Peralta CA, Shlipak MG, Judd S, et al. Detection of chronic kidney disease with creatinine, cystatin $\mathrm{C}$, and urine albumin-to-creatinine ratio and association with progression to end-stage renal disease and mortality. JAMA. 2011;305(15):1545-1552.

59. Shlipak MG, Matsushita K, Arnlov J, et al; CKD Prognosis Consortium. Cystatin $\mathrm{C}$ versus creatinine in determining risk based on kidney function. N Engl J Med. 2013;369(10):932-943.

60. Blakeman T, Protheroe J, Chew-Graham C, Rogers A, Kennedy A. Understanding the management of early-stage chronic kidney disease in primary care: a qualitative study. $\mathrm{Br} J$ Gen Pract. 2012;62(597):e233-e242.

61. Crinson I, Gallagher H, Thomas N, de Lusignan S. How ready is general practice to improve quality in chronic kidney disease? A diagnostic analysis. Br J Gen Pract. 2010;60(575):403-409.

62. Blood Pressure Lowering Treatment Trialists' Collaboration; Ninomiya T, Perkovic V, et al. Blood pressure lowering and major cardiovascular events in people with and without chronic kidney disease: meta-analysis of randomised controlled trials. BMJ. 2013;347:f5680.

63. Plantinga LC, Boulware LE, Coresh J, et al. Patient awareness of chronic kidney disease: trends and predictors. Arch Intern Med. 2008;168(20):2268-2275.

64. Slevin J, Taylor A. Understanding what the public know about their kidneys and what they do: Findings from Ipsos MORI survey - July 2014. In: NHS England and UK Renal Registry 'Think Kidneys' Programme 2015, London, England.

65. Armstrong D. Diagnosis and nosology in primary care. Soc Sci Med. 2011;73(6):801-807.

66. Moynihan R, Glassock R, Doust J. Chronic kidney disease controversy: how expanding definitions are unnecessarily labelling many people as diseased. BMJ. 2013;347:f4298.

67. Daker-White G, Rogers A, Kennedy A, Blakeman T, Blickem C, ChewGraham C. Non-disclosure of chronic kidney disease in primary care and the limits of instrumental rationality in chronic illness self-management. Soc Sci Med. 2015;131:31-39.

68. Gaffney H, Blakeman T, Blickem C, et al. Predictors of patient selfreport of chronic kidney disease: baseline analysis of a randomised controlled trial. BMC Fam Pract. 2014;15:196.

69. Greer RC, Cooper LA, Crews DC, Powe NR, Boulware LE. Quality of patient-physician discussions about CKD in primary care: a crosssectional study. Am J Kidney Dis. 2011;57(4):583-591.

70. Blakeman T, Blickem C, Kennedy A, et al. Effect of information and telephone-guided access to community support for people with chronic kidney disease: randomised controlled trial. PLoS One. 2014;9(10):e109135.
71. Simmonds R, Evans J, Feder G, et al. Understanding tensions and identifying clinician agreement on improvements to early-stage chronic kidney disease monitoring in primary care: a qualitative study. $B M J$ Open. 2016;6(3):e010337.

72. KDIGO Acute Kidney Injury Work Group. KDIGO clinical practice guideline for acute kidney injury. Kidney Int Suppl. 2012;2:138.

73. Tangri N, Kitsios GD, Inker LA, et al. Risk prediction models for patients with chronic kidney disease: a systematic review. Ann Intern Med. 2013;158(8):596-603.

74. Tangri N, Stevens LA, Griffith J, et al. A predictive model for progression of chronic kidney disease to kidney failure. JAMA. 2011;305(15): 1553-1559.

75. Taal MW. Progress in risk prediction for people with chronic kidney disease. Curr Opin Nephrol Hypertens. 2014;23(6):519-524.

76. Gerstein HC, Mann JF, Yi Q, et al; HOPE Study Investigators. Albuminuria and risk of cardiovascular events, death, and heart failure in diabetic and nondiabetic individuals. JAMA. 2001;286(4): 421-426.

77. James MT, Grams ME, Woodward M, et al; Prognosis Consortium. A meta-analysis of the association of estimated GFR, albuminuria, diabetes mellitus, and hypertension with acute kidney injury. $A m J$ Kidney Dis. 2015;66(4):602-612.

78. Yuyun MF, Khaw KT, Luben R, et al. A prospective study of microalbuminuria and incident coronary heart disease and its prognostic significance in a British population: the EPIC-Norfolk study. Am J Epidemiol. 2004;159(3):284-293.

79. Fraser SD, Roderick PJ, McIntyre NJ, et al. Suboptimal blood pressure control in chronic kidney disease stage 3: baseline data from a cohort study in primary care. BMC Fam Pract. 2013;14:88.

80. Kalaitzidis R, Li S, Wang C, Chen S-C, McCullough PA, Bakris GL. Hypertension in early-stage kidney disease: an update from the kidney early evaluation program (KEEP). Am J Kidney Dis. 2009;53(4): S22-S31.

81. Gallagher H, de Lusignan S, Harris K, Cates C. Quality-improvement strategies for the management of hypertension in chronic kidney disease in primary care: a systematic review. Br J Gen Pract. 2010;60(575): e258-e265.

82. Lewis JB. Blood pressure control in chronic kidney disease: is less really more? J Am Soc Nephrol. 2010;21(7):1086-1092.

83. de Lusignana S, Gallagher H, Jones S, et al. Audit-based education lowers systolic blood pressure in chronic kidney disease: the quality improvement in CKD (QICKD) trial results. Kidney Int. 2013;84(3): 609-620.

84. Sanghavi S, Vassalotti JA. Practical use of home blood pressure monitoring in chronic kidney disease. Cardiorenal Med. 2014;4(2): $113-122$.

85. Hill NR, Lasserson D, Thompson B, et al. Benefits of Aldosterone Receptor Antagonism in Chronic Kidney Disease (BARACK D) trial-a multi-centre, prospective, randomised, open, blinded end-point, 36-month study of 2,616 patients within primary care with stage $3 \mathrm{~b}$ chronic kidney disease to compare the efficacy of spironolactone $25 \mathrm{mg}$ once daily in addition to routine care on mortality and cardiovascular outcomes versus routine care alone: study protocol for a randomized controlled trial. Trials. 2014;15:160.

86. Chang A, Kramer H. Should eGFR and albuminuria be added to the Framingham risk score? Chronic kidney disease and cardiovascular disease risk prediction. Nephron Clin Pract. 2011;119(2):c171-c177. [discussion c177-178].

87. Hippisley-Cox J, Coupland C, Robson J, Brindle P. Derivation, validation, and evaluation of a new QRISK model to estimate lifetime risk of cardiovascular disease: cohort study using QResearch database. BMJ. 2010;341:c6624.

88. NICE Clinical Guidelines 181. Cardiovascular Disease: Risk Assessment and Reduction, Including Lipid Modification. London: National Institute of Health and Care Excellence; 2014.

89. Wang HE, Muntner P, Chertow GM, Warnock DG. Acute kidney injury and mortality in hospitalized patients. Am J Nephrol.2012;35(4):349-355. 
90. Blakeman T, Harding S, O’Donoghue D. Acute kidney injury in the community: why primary care has an important role. Br J Gen Pract. 2013;63(609):173-174.

91. Jansen DL, Heijmans MJ, Rijken M, et al. Illness perceptions and treatment perceptions of patients with chronic kidney disease: different phases, different perceptions? Br J Health Psychol. 2013;18(2):244-262.

92. Valentijn PP, Schepman SM, Opheij W, Bruijnzeels MA. Understanding integrated care: a comprehensive conceptual framework based on the integrative functions of primary care. Int J Integr Care. 2013;13:e010.

93. Calestani M, Tonkin-Crine S, Pruthi R, et al; ATTOM Investigators. Patient attitudes towards kidney transplant listing: qualitative findings from the ATTOM study. Nephrol Dial Transplant. 2014;29(11):2144-2150.

94. Fraser SD, Roderick PJ, Casey M, Taal MW, Yuen HM, Nutbeam D. Prevalence and associations of limited health literacy in chronic kidney disease: a systematic review. Nephrol Dial Transplant. 2013;28(1):129-137.
95. McIntyre NJ, Fluck R, McIntyre C, Taal M. Treatment needs and diagnosis awareness in primary care patients with chronic kidney disease. Br J Gen Pract. 2012;62(597):e227-e232.

96. Taal MW. Chronic kidney disease in general populations and primary care: diagnostic and therapeutic considerations. Curr Opin Nephrol Hypertens. 2013;22(6):593-598.

97. Tsang JY, Blakeman T, Hegarty J, Humphreys J, Harvey G. Understanding the implementation of interventions to improve the management of chronic kidney disease in primary care: a rapid realist review. Implement Sci. 2016;11(1):47.

98. Barahimi H,Abolhassani F, Rajaee F, Akbari Sari A. A multilevel model for services provided to patients with chronic kidney disease. Iran JKidney Dis. 2015;9(3):186-192.

99. WHO. The World Health Report 2008 - Primary Health Care (Now More Than Ever). Geneva: World Health Organisation; 2008.
Pragmatic and Observational Research

\section{Publish your work in this journal}

Pragmatic and Observational Research is an international, peer-reviewed, open access journal that publishes data from studies designed to reflect more closely medical interventions in real-world clinical practice compared with classical randomized controlled trials (RCTs). The manuscript management system is completely online and includes a very quick and fair peer-review
Dovepress

system. Visit http://www.dovepress.com/testimonials.php to read real quotes from published authors. 